\title{
THE PERIOD-LUMINOSITY RELATION FOR \\ CEPHEIDS IN GLOBULAR CLUSTERS
}

\author{
B. V. KUKARKIN and A. S. RASTORGOUEV \\ Sternberg Astronomical Institute, U.S.S.R.
}

The period-luminosity relation for Cepheids in globular clusters has been investigated many times (e.g. Fernie, 1964; Kwee, 1968; Frolov, 1970; Demers, 1971).

The method of determination of the apparent distance moduli was recently revised by Kukarkin and Russev (1972). Instead of using a single absolute magnitude for RR Lyrae variables, the magnitudes according to pulsation theory (Christy, 1966, 1971) were adopted. The inhomogeneity of the absolute magnitudes of the RR Lyrae variables had already been established long ago (Pavlovskaya, 1953), but it attracted attention only recently. The different methods for determining the distance moduli of globular clusters were calibrated according to the new absolute magnitudes of the RR Lyrae variables. The problem consisted in the determination of the absolute magnitudes of the Cepheids in globular clusters according to the apparent distance moduli.

The photographic observations of 9 Cepheids in the globular clusters M5, M10, M12, M13 and M80 were made by A. S. Rastorgouev in the $\boldsymbol{B}$ photometric system on

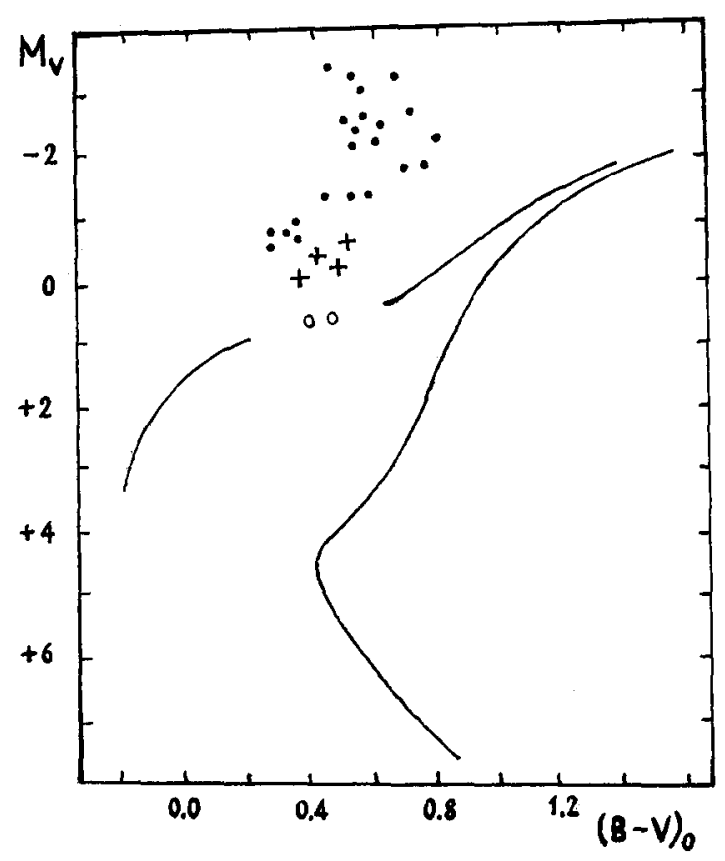

Fig. 1. Location of globular cluster Cepheids in the colour-magnitude away. Crosses represent peculiar Cepheids. 
plates obtained at our Crimean Station. The results are given in Table I. (The observations will be published separately).

All the observations of Cepheids in globular clusters by different authors were reduced to the $B V$ photometric system. The results are given in Table II. In the first column the NGC number of the globular cluster is given; in the second the number of

TABLE I

Crimean photographic observations

\begin{tabular}{cccc}
\hline NGC & Var & $\log P$ & $B$ \\
\hline 6205 & V1 & 0.164 & 15.10 \\
6205 & V6 & 0.325 & 14.40 \\
6205 & V2 & 0.708 & 13.20 \\
6254 & V3 & 0.896 & 13.80 \\
6218 & V1 & 1.190 & 12.72 \\
6093 & V1 & 1.212 & 14.10 \\
6254 & V2 & 1.273 & 12.65 \\
5904 & V42 & 1.411 & 11.90 \\
5904 & V 84 & 1.423 & 12.05 \\
\hline
\end{tabular}

TABLE II

Reduced photographic observations

\begin{tabular}{|c|c|c|c|c|c|c|c|c|c|}
\hline NGC & Var & $\log P$ & $\langle V\rangle$ & $\langle\boldsymbol{B}\rangle$ & $\langle B-V\rangle$ & $\langle B-V\rangle_{0}$ & $\bmod _{V}$ & $M_{V}$ & $M_{B}$ \\
\hline 5139 & 43 & 0.063 & $13^{m} \cdot 38$ & $13 \div 84$ & $0^{\mathrm{m}} \cdot 46$ & $0 \div 32$ & $13^{m} \div 84$ & $-0^{\mathrm{m}} \cdot 46$ & $-0 \div 14$ \\
\hline 5139 & 92 & 0.129 & 13.96 & 14.45 & 0.49 & 0.35 & 13.84 & +0.12 & +0.47 \\
\hline 5139 & 60 & 0.130 & 13.49 & 13.87 & 0.38 & 0.24 & 13.84 & -0.35 & -0.11 \\
\hline 7078 & 1 & 0.158 & 14.85 & 15.17 & 0.34 & 0.23 & 15.30 & -0.47 & -0.24 \\
\hline 6205 & 1 & 0.164 & 14.19 & 14.49 & 0.41 & 0.38 & 13.93 & -0.15 & +0.23 \\
\hline 6402 & 76 & 0.276 & 15.84 & 16.60 & 0.76 & 0.27 & 16.33 & -0.49 & -0.22 \\
\hline 6205 & 6 & 0.325 & 13.89 & 14.39 & 0.50 & 0.47 & 13.93 & -0.04 & +0.43 \\
\hline 5139 & 61 & 0.357 & 13.44 & 14.07 & 0.63 & 0.49 & 13.84 & -0.40 & +0.09 \\
\hline 6402 & 2 & 0.445 & 15.64 & 16.44 & 0.80 & 0.31 & 16.33 & -0.69 & -0.38 \\
\hline 5139 & 48 & 0.651 & 12.77 & 13.41 & 0.64 & 0.50 & 13.84 & -1.07 & -0.57 \\
\hline 6205 & 2 & 0.708 & 12.86 & 13.28 & 0.42 & 0.39 & 13.93 & -1.07 & -0.68 \\
\hline 6254 & 3 & 0.896 & 12.80 & 13.59 & 0.79 & 0.56 & 13.92 & -1.12 & -0.56 \\
\hline 6402 & 17 & 1.083 & 14.79 & 15.93 & 1.14 & 0.65 & 16.33 & -1.54 & -0.89 \\
\hline 6402 & 7 & 1.133 & 14.75 & 15.97 & 1.22 & 0.73 & 16.33 & -1.58 & -0.85 \\
\hline 5139 & 29 & 1.168 & 11.88 & 12.83 & 0.95 & 0.81 & 13.84 & -1.96 & -1.15 \\
\hline 5272 & 154 & 1.185 & 12.48 & 12.97 & 0.49 & 0.47 & 14.82 & -2.34 & -1.87 \\
\hline 6218 & 1 & 1.190 & - & 12.72 & - & - & 14.09 & - & -1.54 \\
\hline 7089 & 1 & 1.192 & 13.42 & 13.99 & 0.57 & 0.50 & 15.44 & -2.02 & -1.52 \\
\hline 6093 & 1 & 1.212 & 13.44 & 14.20 & 0.76 & 0.58 & 15.45: & $-2.01:$ & -1.43 \\
\hline 7089 & 5 & 1.244 & 13.29 & 13.86 & 0.57 & 0.50 & 15.44 & -2.15 & -1.65 \\
\hline 6402 & 1 & 1.272 & 14.09 & 15.31 & 1.22 & 0.73 & 16.33 & -2.24 & -1.51 \\
\hline 6254 & 2 & 1.273 & 11.74 & 12.56 & 0.82 & 0.59 & 13.92 & -2.18 & -1.59 \\
\hline 7089 & 6 & 1.284 & 13.20 & 13.70 & 0.60 & 0.53 & 15.44 & -2.34 & -1.81 \\
\hline 5904 & 42 & 1.411 & 11.28 & 11.84 & 0.56 & 0.51 & 14.33 & -3.05 & -2.54 \\
\hline 5904 & 84 & 1.423 & 11.42 & 12.01 & 0.59 & 0.54 & 14.33 & -2.91 & -2.37 \\
\hline 5139 & 1 & 1.465 & 10.89 & 11.68 & 0.79 & 0.65 & 13.84 & -2.95 & -2.30 \\
\hline 7089 & 11 & 1.525 & 12.21 & 12.72 & 0.51 & 0.44 & 15.44 & -3.23 & -2.79 \\
\hline
\end{tabular}


the variable according to the catalogue of Sawyer Hogg (1955); in the third the logarithm of the period; in the fourth and fifth the apparent mean magnitudes $\langle V\rangle$ and $\langle B\rangle$; in the sixth the value $\langle B-V\rangle$; in the seventh $\langle B-V\rangle_{0}$; in the eighth the apparent distance modulus $\bmod _{V}$; in the ninth and tenth the absolute magnitudes $M_{V}$ and $M_{B}$.

In Figure 1 the positions of the Cepheids on the colour-magnitude diagram are given. The instability strip of the Cepheids in globular clusters is slightly different from those of the Classical Cepheids. Four Cepheids indicated by crosses differ from the other Cepheids in some relations (see e.g. Figure 4).

In Figures 2 and 3 the period-luminosity relations in $V$ and $B$ are given. The abovementioned four Cepheids are again shown by crosses. The peculiarity of these Cepheids is illustrated by the example of the variables V 60 and V 92 in the globular cluster $\omega$ Centauri. Figure 4 gives the light curves of these variables (Martin, 1938). The curve of V 60 is very similar to those of other Cepheids with the same period. The curve of $\mathrm{V} 92$ is very different!

It is possible that these four Cepheids are in phases of evolution different to the majority of Cepheids in globular clusters (Schwarzschild, 1970).

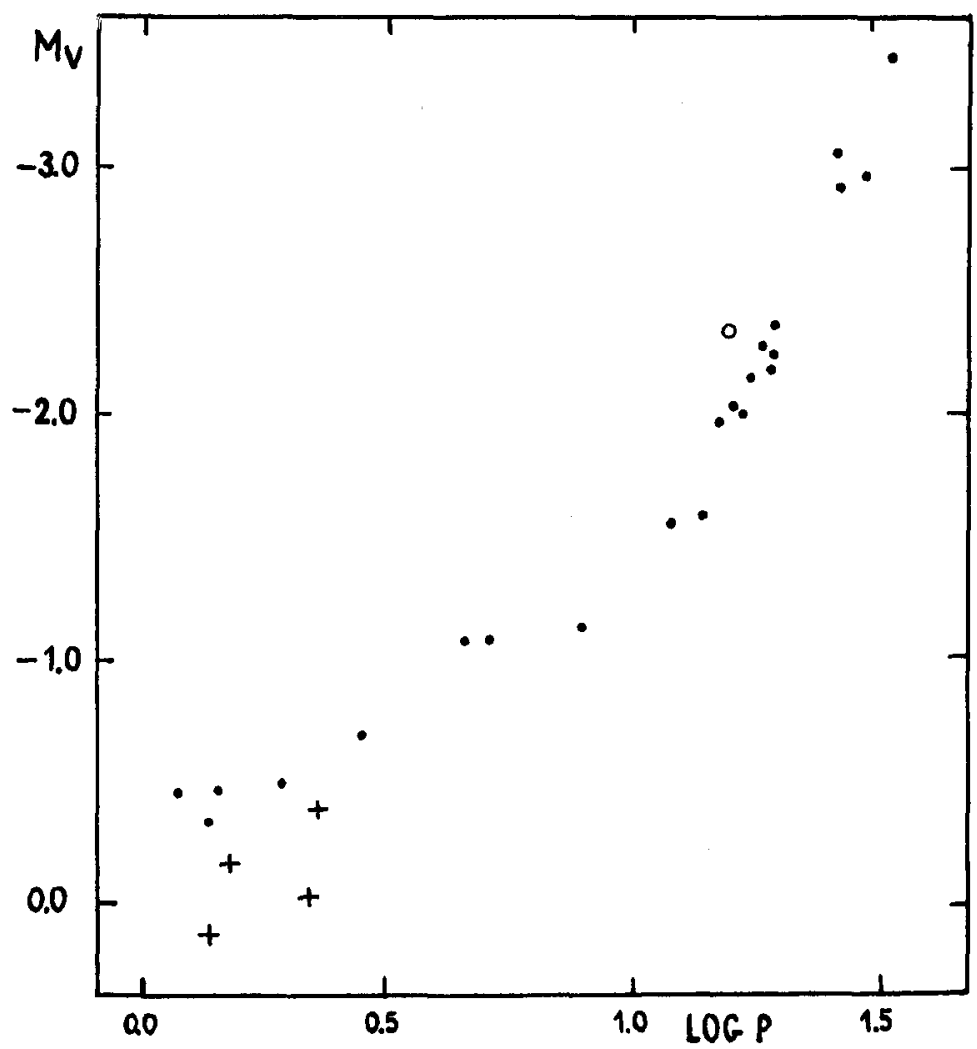

Figs. 2. and 3. The period-luminosity relations for globular cluster Cepheids. 


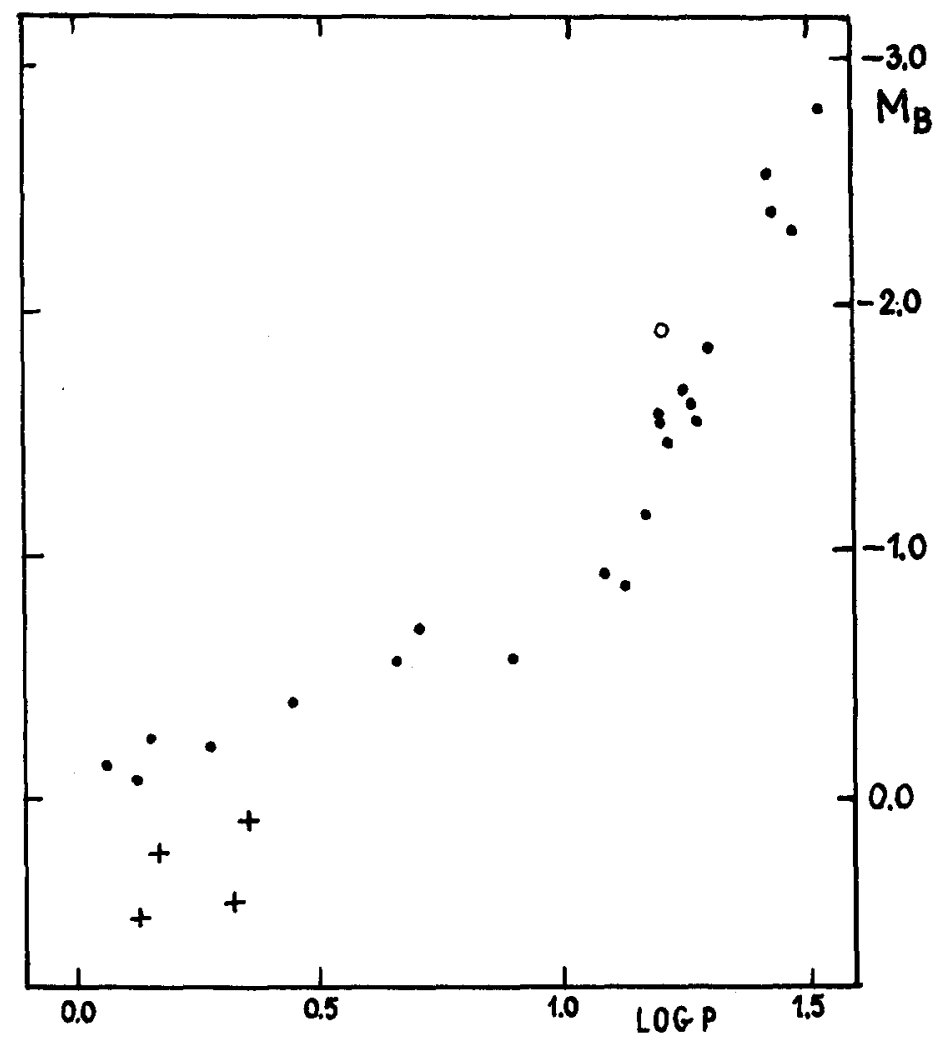

Fig. 3.

The period-luminosity relation of the Cepheids in globular clusters may be represented by the following linear equations:

$$
\left.\begin{array}{rlr}
M_{V}= & -0.26-1.12 \log P & (\log P<1.14) \\
& \pm 0.07 \pm 0.08 & \\
M_{V}= & +2.66-3.89 \log P & (\log P>1.14) \\
& \pm 0.10 \pm 0.11 & \\
M_{B}= & -0.08-0.70 \log P \quad(\log P<1.14) \\
& \pm 0.06 \pm 0.07 & \\
M_{B}= & -3.51+4.11 \log P \quad(\log P>1.14) \\
& \pm 0.09 \pm 0.08 &
\end{array}\right\}
$$

The problem of the period-luminosity relation for the Cepheids in globular clusters is complicated. About $85 \%$ of Cepheids form a single physical group, but $15 \%$ have peculiarities. When using Equations (1) and (2) it is necessary to take into account not only the periods, but also the shape of the light curve and other properties. 


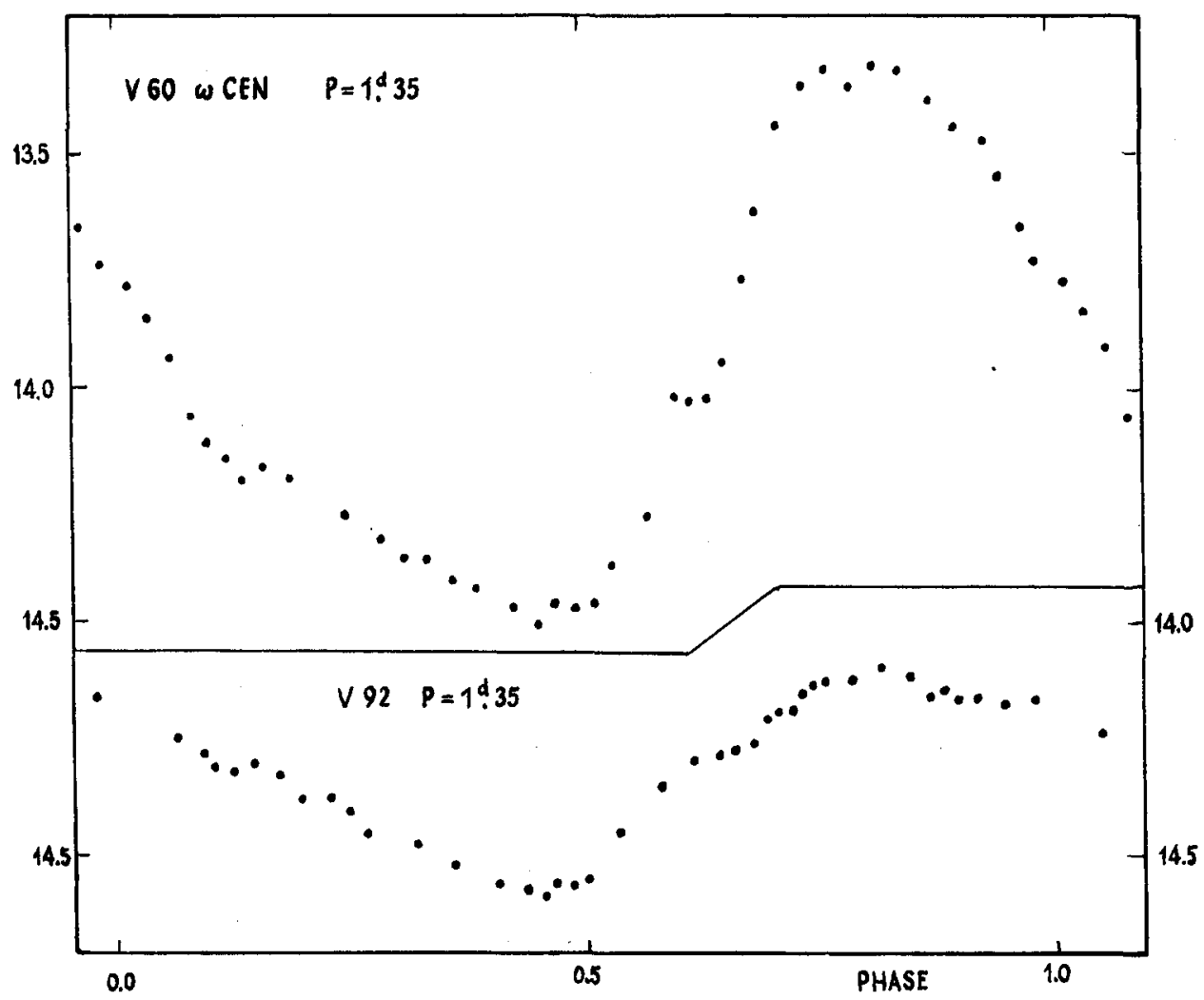

Fig. 4. Differing light-curves of two Cepheids of equal pei iod in $\omega$ Cen.

\section{References}

Christy, R. F.: 1966, 144, 108.

Christy, R. F.: 1971, in Highlights of Astronomy, Vol. 2, D. Reidel, Dordrecht-Holland, p. 777.

Demers, S.: 1971, Astron. J. 76, 916.

Fernie, J. D.: 1964, Astron. J. 69, 258.

Frolov, M.S.: 1970, in B. V. Kukarkina, (ed.), Pulsating Stars; Non-stationary Stars and Methods of their Investigation, Moscow, Izdatel'stvo 'Nauka'. Glavnaya Redaktsiya Fiziko - Matematchesikoy Literatury, p. 124.

Kukarkin, B. V. and Russev, R. M.: 1972, Astron. Zh. 49, 121.

Kwee, K. K.: 1968, Bull. Astron. Inst. Netherlands 19, 374.

Martin, W. C.: 1938, Ann. Sterrew. Leiden 17, No. 2

Pavlovskaya, E. D.: 1953, Perem. Zvezdy 9, 349.

Schwarzschild, M. and Härm, R.: 1970, Astrophys. J. 160, 341. 